\title{
Ischaemic Monomelic Neuropathy in a Diabetic Patient after Brachio-Basilic Arteriovenous Fistula Creation
}

\author{
Nicholas B. Shannon"1, Siew Ping Chng², Chieh Suai Tan², Chin-Ann Johnny Ong² \\ ${ }^{1}$ Duke-NUS Graduate Medical School, Singapore City, Singapore \\ ${ }^{2}$ Singapore General Hospital, Singapore City, Singapore \\ Email: johnny.ong.c.a@sgh.com.sg
}

Received 14 Mayy 2016; accepted 12 June 2016; published 15 June 2016

Copyright (C) 2016 by authors and Scientific Research Publishing Inc.

This work is licensed under the Creative Commons Attribution International License (CC BY).

http://creativecommons.org/licenses/by/4.0/

(c) (i) Open Access

\begin{abstract}
Introduction: The number of people with diabetes is increasing in every country. Recent estimates put the prevalence at $8.3 \%$ of adults, with a predicted rise to 10.1 in 2035 [1]. Although less than $1 \%$ of patients with diabetes require definitive haemodialysis, this still represents a significant number who may require arteriovenous fistula creation. Presentation: We report a case of IMN occurring in a patient with end stage diabetic nephropathy following brachio-basilic arteriovenous fistula creation for chronic maintenance haemodialysis. Discussion: Ischaemicmonomelic neuropathy (IMN) has developed as a distinct clinical entity involving dysfunction of multiple peripheral nerves following vascular access. Symptom onset is usually immediate, and neurological symptoms are dominant, generally in the absence of significant clinical ischaemia. Acute neurological symptoms may be disabling and irreversible, but prompt ligation of arteriovenous fistula can prevent permanent disability. Conclusion: Ischaemicmonomelic neuropathy (IMN) is a rare but serious complication of vascular access for arteriovenous fistula for haemodialysis and should be considered in the differential diagnosis of hand dysfunction following such surgery. While the condition is not preventable or predictable, prompt recognition and treatment can lead to prevention of permanent disability.
\end{abstract}

\section{Keywords}

Diabetes, Vascular, Neuropathy

\section{Introduction}

The number of people with diabetes is increasing in every country. Recent estimates put the prevalence at $8.3 \%$

How to cite this paper: Shannon, N.B., Chng, S.P., Tan, C.S. and Ong, C.-A.J. (2016) Ischaemic Monomelic Neuropathy in a Diabetic Patient after Brachio-Basilic Arteriovenous Fistula Creation. Case Reports in Clinical Medicine, 5, $199-202$. 
of adults, with a predicted rise to $10.1 \%$ in 2035 [1]. Although less than $1 \%$ of patients with diabetes require renal replacement therapy, this still represents a significant number who may require arteriovenous fistula creation.

We highlight ischaemicmonomelic neuropathy (IMN) as a rare but serious complication which should be considered in the differential diagnosis of hand dysfunction following vascular access. While the condition is not preventable or predictable, prompt recognition and treatment is essential, and can prevent permanent disability from rapid onset nerve damage.

\section{Presentation of Case}

A patient with diabetes mellitus presented with shortness of breath, abdominal distension and lower limb swelling for one week due to acute kidney injury secondary to sepsis from infection of a poorly healing wound post right patella fracture repair six months prior to presentation. Additional past history was notable for poorly controlled diabetes, hyperlipidaemia, anaemia of chronic disease, and chronic renal impairment. Surgical history was significant for an infected left toe ulcer for which the patient underwent debridement. On examination, blood pressure was $148 / 85$, and pulse rate was 82 /minute and regular. Jugular venous pressure was noted to be elevated and bilateral pedal oedema was present up to the knees. Coarse crepitations were noted in the bases of both lungs and gross ascites was present. Laboratory investigations revealed a haemoglobin level of 9.7 [14.0 18.0 G/DL] with a while cell count of 11.33 [4.0 $\left.-10.0 \times 10^{9} / \mathrm{L}\right]$. Platelet count was $214\left[140-440 \times 10^{9} / \mathrm{L}\right]$. Serum creatinine was $376[54-101 \mu \mathrm{Mol} / \mathrm{L}]$, and serum urea, bicarbonate and potassium levels were 17.2 [2.7 $6.9 \mu \mathrm{Mol} / \mathrm{L}], 20.1[19.0-29.0 \mu \mathrm{Mol} / \mathrm{L}]$ and $3.8[3.6-5.0 \mu \mathrm{Mol} / \mathrm{L}]$ respectively. Chest $\mathrm{x}$-ray was consistent with acute pulmonary oedema. The patient was started on diuretics including intravenous furosemide and oral metalazone. Hemodialysis was commenced via a tunnelled vascular catheter and planned for the creation of an arterio-venous fistula as a form of a permanent access for dialysis.

79 days after initial admission, a brachio-basilic arteriovenous fistula was created in the ambulatory surgical unit. Within hours after the surgery, the patient developed numbness of the right $4^{\text {th }}$ and $5^{\text {th }}$ fingers and thumb. The right upper limb was swollen, with reduced range of motion of the right wrist and fingers. The patient was unable to make a fist and exhibited clawing (Figure 1). Radial pulses were present bilaterally, capillary refill was $<2$ seconds and the hands were noted to be warm. The neurological deficits persisted over the next two hours and were noted to be worsening as reported by the patient.

A clinical diagnosis of IMN with signs of sensorimotoric dysfunction of the nerves of the hand was made based on neurological symptoms out of proportion to physical findings, good pulse and warm peripheries. Reoperation with ligation of the AVF was performed within 24 hours of the fistula creation. Post-operatively an improvement in sensation and range of motion of the limb was noted. Over the next couple of weeks a complete recovery was made.

\section{Discussion}

Differential diagnosis of hand dysfunction following AV access include neurological complications of axillary

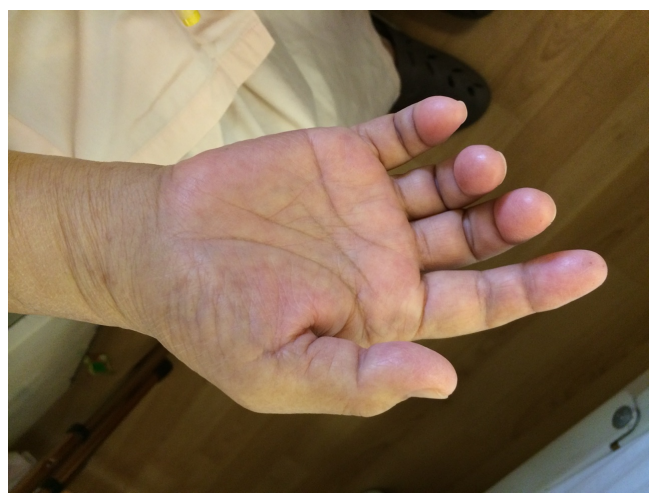

Figure 1. Photograph of right hand demonstrating clawing and absence of ischaemic pallor. 
block or as a result of incorrect positioning during surgery, peripheral nerve compression as a result of acute compartment syndrome, functional deficit secondary to surgical trauma, postoperative pain or postoperative swelling. Vascular differentials include arterial emboli or thrombi, vascular steal syndrome, and ischaemicmonomelic neuropathy. In vascular steal syndrome there is distal ischaemia, manifesting as numbness, pain and paraesthesia. Clinical findings include reduced peripheral temperatures, diminished or absent pulses, and delayed capillary refill. Reduced digital pressure and absent Doppler signal can be used to support the diagnosis. Symptoms may occur immediately after vascular access, but can also be delayed, and may develop up to several months later.

Ischaemicmonomelic neuropathy (IMN) has developed as a distinct clinical entity involving dysfunction of multiple peripheral nerves. Symptom onset is usually immediate, and neurological symptoms are dominant, generally in the absence of significant clinical ischaemia. Typically the hand is warm, pulses are present, and capillary refill is preserved. Mildly reduced or normal digital pressures and audible Doppler signal can support the diagnosis. Features supporting IMN over a vascular steal syndrome are brachial artery based fistula, presence of a radial pulse, and limited evidence of clinical ischaemia. Positive arterial Doppler studies and normal or slightly decreased digital pressures can aid in differentiating between the 2 conditions.

An initial description of IMN was made by Bolton et al. in 1979 [2], but it was not until 1983 when Wilbourn et al. formalised the term IMN [3]. The initial report described a clinical entity with arterial insufficiency (ischaemic), involving a single extremity (monomelic) and causing selective dysfunction (neuropathy) of multiple peripheral nerves. In his subsequent report, Wilbourn described three patients, two with acute arterial occlusions, and one with creation of an AVF for permanent access for hemodialysis. Following this Riggs et al. in 1989 described upper extremity IMN occurring after shunt placement in diabetic patients with end stage renal disease and evidence of peripheral vascular disease [4]. The pathophysiology of IMN has been attributed to a decrease in arterial blood flow secondary to diversion of large amount of blood away from the distal forearm and hand following arterio-venous shunt formation in the proximal forearm, or as a result of acute noncompressive major arterial occlusion. Under-perfusion of vasa nervosum results in acute axonal loss in multiple peripheral nerve fibres. Acute neurological symptoms may be disabling and irreversible, but there is not sufficient ischaemia to cause muscle or skin necrosis. It is thought that previous neuropathy results in a lower threshold to ischaemic injury leading to an increased risk of IMN in patients with diabetes mellitus, atherosclerotic peripheral vascular disease and women [5]-[8]. Raheb et al. reviewed 12 cases of IMN that occurred in 273 patients (4.4\%) undergoing placement of PTFE brachial artery based loop graph and reported that all patients who developed IMN were diabetic and female [9].

Brachial artery based vascular access is most commonly associated with IMN due to lack of collateral blood supply to the distal arm. Zanow et al. reviewed a personal experience of 4853 procedures and found the incidence of ischaemia in patients with arteriovenous access to be $0.3 \%$ for wrist fistulas, $1.8 \%(0.9 \%-5.2 \%)$ for elbow fistulas and 2.2\% for upper extremity PTFE grafts [10].

Electrophysiological findings can be used to confirm a diagnosis and include axonal loss, low amplitude or absent responses to sensory and motor nerve stimulation with relatively preserved conduction velocities, and fibrillations and reduced motor unit requirement on needle EMG, but treatment should not be delayed when clinical suspicion is high. Kaku et al. reported in 1993 that conduction block occurs early in the course of upper extremity IMN, but this may not be present later in the disease [11].

Prognosis depends largely on the amount of initial nerve damage. Functional improvement may be seen as the nerve regenerates at a rate of approximately $2.5 \mathrm{~cm}$ per month. In the hand disability may result from weakness of the thenar and intrinsic muscles as well as sensory loss in all fingers which may extend into the palm and hand. Contractures of joints may also develop. For some patients pain is the most disabling aspect of this disease.

In the case presented here a clinical diagnosis of IMN with signs of sensorimotoric dysfunction of the nerves of the hand was made based on neurological symptoms out of proportion to physical findings, good pulse and warm peripheries. Based on high clinical suspicion and the urgency of treatment further electrophysiological testing was not carried out to avoid any delay in treatment. Reoperation with ligation of the AVF was performed within 24 hours of the fistula creation. Post-operatively an improvement in sensation and range of motion of the limb was noted. Over the next couple of weeks a complete recovery was made. Although permanent damage can result as a result of IMN, in this patient early reoperation to ligate the fistula allowed complete recovery within a month. This case highlights the importance of prompt diagnosis and treatment in this condition as well as hig- 
hlighting the differential in patients with diabetes undergoing vascular access given the increasing number of such patients.

\section{Conclusion}

The distinction between vascular steal syndrome and IMN is important as vascular steal syndrome and is readily treated with access revision while treatment of IMN requires immediate closure of the AVF (NKF K/DOQI 2006 guideline 5.6). In Singapore, the rate of new diagnoses of diabetes was increasing, currently affecting 11.3\% of the population, up from 8.2\% in 2004 [12]. It is estimated that by 2030, 14\% - 15\% of the adult Singaporean population will be diabetic [13]. Moreover, diabetic nephropathy (DN) is the main cause of Stage 5 Chronic Kidney Disease (CKD 5) in Singapore. Haemodialysis is the main dialysis modality and 86.6\% of incident CKD 5 patients in 2014 are on hemodialysis [1]. Arteriovenous fistula is the preferred vascular access modality and with the increasing incidence of stage 5 CKD secondary to DM nephropathy, the incidence of ischaemicmonomelic neuropathy may increase with increased number of haemodialysis vascular access creation. It is critical that IMN is considered in the differential diagnosis of hand dysfunction following vascular access as it is potentially reversible and prompt recognition and treatment can lead to prevention of permanent disability.

\section{References}

[1] Singapore Renal Registry (2015) National Registry of Diseases Office, Ministry of Health, Singapore.

[2] Bolton, C.F., Driedger, A.A. and Lindsay, R.M. (1979) Ischaemic Neuropathy in Uraemic Patients Caused by Bovine Arteriovenous Shunt. Journal of Neurology, Neurosurgery \& Psychiatry, 42, 810-814. http://dx.doi.org/10.1136/jnnp.42.9.810

[3] Wilbourn, A.J., Furlan, A.J., Hulley, W. and Ruschhaupt, W. (1983) Ischemic Monomelic Neuropathy. Neurology, 33, 447-451. http://dx.doi.org/10.1212/WNL.33.4.447

[4] Riggs, J.E., Moss, A.H., Labosky, D.A., et al. (1989) Upper Extremity Ischemic Monomelic Neuropathy: A Complication of Vascular Access Procedures in Uremic Diabetic Patients. Neurology, 39, 997-998. http://dx.doi.org/10.1212/WNL.39.7.997

[5] Hye, R.J. and Wolf, Y.G. (1994) Ischemic Monomelic Neuropathy: An Under-Recognized Complication of Hemodialysis Access. Annals of Vascular Surgery, 8, 578-582. http://dx.doi.org/10.1007/BF02017415

[6] Wodicka, R. and Isaacs, J. (2010) Ischemic Monomelic Neuropathy. Journal of Hand Surgery, 35, 842-843. http://dx.doi.org/10.1016/j.jhsa.2009.08.014

[7] Kirksey, L. (2010) Ischemic Monomelic Neuropathy: An Underappreciated Cause of Pain and Disability Following Vascular Access Surgery. The Journal of Vascular Access, 11, 165-168.

[8] Redfern, A.B. and Zimmerman, N.B. (1995) Neurologic and Ischemic Complications of upper Extremity Vascular Access for Dialysis. Journal of Hand Surgery, 20, 199-204. http://dx.doi.org/10.1016/S0363-5023(05)80007-3

[9] Raheb, J.R., Esterl, R., Reuter, R., et al. (2001) Ischemic Monomelic Neuropathy as a Complication of Forearm PTFE Loop Grafts in Uremic Diabetic Patients. Vascular Access for Hemodialysis VII, Gore, Chicago, 193-199.

[10] Zanow, J., Petzold, M., Petzold, K., et al. (2001) Diagnosis and Differentiated Treatment of Ischemia in Patients with Arteriovenous Vascular Access. Vascular Access for Hemodialysis-VII, Gore, Chicago, 201-209.

[11] Kaku, D.A., Malamut, R.I., Frey, D.J. and Gareth, J.P. (1993) Conduction Block as an Early Sign of Reversible Injury in Ischemic Monomelic Neuropathy. Neurology, 43, 1126-1130. http://dx.doi.org/10.1212/WNL.43.6.1126

[12] Diabetes Mellitus. National Health Survey 2010 (2011) Singapore: Epidemiology \& Disease Control Division, Ministry of Health.

[13] Toh, Y.C., Yeo, G., Sidek, I. and Tan, H.B. (2006) Feedback Ideas-In Focus. REACH, Singapore. 